\title{
Prevalence and risk factors of periodontitis among adults with or without diabetes mellitus
}

\author{
Mihee Hong, Hee Yeon Kim, Hannah Seok, Chang Dong Yeo, Young Soo Kim, Jae Yen Song, \\ Young Bok Lee, Dong-Hee Lee, Jae-Im Lee, Tae-Kyu Lee, Hyo-Suk Ahn, Yoon Ho Ko, \\ Seong Cheol Jeong, Hiun Suk Chae, and Tae Seo Sohn
}

Epidemiology Study Cluster of Uijeongbu St. Mary's Hospital, College of Medicine, The Catholic University of Korea, Seoul, Korea

Received: December 28, 2015

Revised : June 13, 2016

Accepted: July 28, 2016

\section{Correspondence to}

\section{Tae Seo Sohn, M.D.}

Department of Internal Medicine, College of Medicine, Uijeongbu St. Mary's Hospital, The Catholic

University of Korea, 271

Cheonbo-ro, Uijeongbu 11765,

Korea

Tel: +82-31-820-3652

Fax: +82-31-847-2719

E-mail:imsts@catholic.ac.kr
Background/Aims: This study examined prevalence and risk factors of periodontitis in representative samples of Korean adults, with and without diabetes mellitus (DM).

Methods: Data from the 2012 Korean National Health and Nutritional Examination Survey were analyzed. A total of 4,477 adults ( $\geq 30$ years old) were selected from 8,057 individuals who completed a nutrition survey, a self-reported general health behavior questionnaire, an oral examination, an oral hygiene behaviors survey, and laboratory tests. DM was defined as a fasting plasma glucose $\geq 126$ $\mathrm{mg} / \mathrm{dL}$, or self-reported diagnosed diabetes, or current use of oral hypoglycemic agents and/or insulin. The community periodontal index was used to assess periodontitis status and comparisons between the periodontitis and the non-periodontitis group, were performed, according to the presence of DM. Risk factors for periodontitis in adults with DM and without DM were evaluated by multiple logistic regression analysis.

Results: The prevalence of periodontitis was significantly higher in adults with DM (43.7\%) than in those without DM (25\%, p< 0.001). In adults without DM, risk factors for periodontitis were older age, male, urban habitation, waist circumference, smoking, oral pain, and less frequent tooth brushing. Significant risk factors for periodontitis in adults with DM were the smoking, oral pain, and not-using an oral hygiene product.

Conclusions: Adults with DM have an increased risk of periodontitis than those without DM. Current smoking and oral pain increase this risk. Using an oral hygiene product can reduce risk of periodontal disease in adults with DM.

Keywords: Periodontitis; Diabetes mellitus; Nutrition surveys; Periodontal diseases; Population surveillance

\section{INTRODUCTION}

Diabetes mellitus (DM) is a group of metabolic disorders characterized by hyperglycemia caused by defects in insulin secretion, insulin action, or both [1]. The adoption of a Westernized lifestyle and the increasing prevalence of obesity have contributed to the increasing prevalence of diabetes in Korea ( $12.4 \%$ of adults $\geq 30$ years old) [2].

Both type 1 and type 2 DM are recognized as major risk factors for periodontitis, resulting in a three-fold increase in susceptibility to periodontitis [3-6]. Periodontal disease with DM is associated with an increased prev- 
alence of periodontal pockets and severe periodontal disease often resulting in unaesthetic and functional problems in dentition $[7,8]$. Recent research concluded that diabetes was positively associated with chronic periodontitis in a representative sample of Korean adults ( $\geq 19$ years old) [9]. The association between DM and periodontitis is established and the presence of one condition increases the risk and severity of the other, and vice versa $[1,5]$.

Periodontitis is a chronic inflammatory disease resulting in the destruction of the connective tissue and bone anchoring teeth to the jaws [10]. Periodontally compromised patients usually present with the maxillary labial segment showing proclinaion, irregular spacing, rotation, and over-eruption of teeth [8]. Furthermore, type 2 DM is associated with a $22 \%$ increased risk of tooth loss [11]. These conditions may require adjunctive or comprehensive orthodontic treatment.

Traditionally, orthodontic treatment was provided for children, adolescents and young adults, usually in their 20s. However, the number of adult patients commencing orthodontic treatment is growing and periodontal disease must be fully controlled before any orthodontic treatment is undertaken, as well as for the duration of treatment and in the long term [12]. In this context, the interaction among risk factors such as age, sex, obesity, and socioeconomic status such as poverty or education, and periodontitis prevalence has received attention $[5,9,13]$. Therefore, we investigated the prevalence and risk factors for periodontitis in adults ( $\geq 30$ years old) with DM and without DM.

\section{METHODS}

\section{Study design and participants}

This study was based on data derived from the fifth Korean National Health and Nutritional Examination Survey (KNHANES), conducted in 2012 by the Korea Center for Disease Control and Prevention. Implemented in 1998, KNHANES is a national project targeting all households and citizens living in the Republic of Korea. The aim is to investigate health status, health awareness, actions to improve health, and food and nutritional intake, of the population providing data for the development and evaluation of health policies and programs in Korea [14].
The subjects were selected using a three-step stratified cluster sampling method from the Population and Housing Census Report. A trained investigator visited each household to complete the questionnaires orally. KHNANES data are used to monitor changes in risk factors and diseases [15].

In $2012,8,057$ individuals out of 10,060 (80\% response rate) participated in the KHNANES survey. Among these, we identified 5,587 individuals aged $\geq 30$ years. Of these, 1,110 were excluded because they did not complete the oral assessment, due to medical conditions or for other reasons. Therefore, a total of 4,477 persons who underwent a complete oral examinations and evaluation of DM status, were included in this study. This study was reviewed and approved by the Institutional Review Board (reference number: 2012-01Exp-01-2C).

$\mathrm{DM}$ in adults aged $\geq 30$ years was defined as follows: fasting plasma glucose $\geq 126 \mathrm{mg} / \mathrm{dL}$ or self-reported diagnosed diabetes or current use of oral hypoglycemic agents and/or insulin [14]. The World Health Organization community periodontal index (CPI) was used to evaluate periodontal health, on a scale ranging from o to 4. The eight molars and the upper right and lower left central incisors were examined and for each sextant and individual, the highest score was recorded as the CPI score [14]. In this study, periodontitis was defined as a CPI value of 3 or 4 , indicating that at least one side had $\mathrm{a} \geq 3.5 \mathrm{~mm}$ pocket.

\section{Study variables}

Definition of the study variables is based on the guidelines for the use of data derived from the fifth KHNANES. Sociodemographic characteristics, including age, sex, education level, household income, habitation (rural), and marital status were extracted from the health interview survey. Variables were defined as follows: age (years), sex (percentage of individuals of male sex for), education level (percentage of individuals completing middle school or higher education), household income (percentage of households on the lowest $25 \%$ quartile of family income), habitation (percentage of subjects living in a rural area), and spouse (percentage of individuals having a spouse).

Nutrition variables, such as daily energy intake (kcal/ day) and protein intake (g/day) were obtained from the nutrition survey data. Systemic conditions, such as hy- 
pertension and hypercholesterolemia, were identified from the health examination data. Hypertension was defined as systolic blood pressure (BP) $\geq 140 \mathrm{mmHg}$, diastolic $\mathrm{BP} \geq 90 \mathrm{mmHg}$ or use of antihypertensive medication. Hypercholesterolemia was defined as fasting total cholesterol (TC) $\geq 240 \mathrm{mg} / \mathrm{dL}$ or use of medication. Data for mental health status was obtained by health interview and the following variables were assessed: stress perception (subjects were asked whether they were under severe stress in their daily life); depression (subjects were asked whether they had feeling of extreme sadness or despair for over 2 weeks in the past year); and suicidal ideation (subjects were asked whether they had thought of committing suicide in the past year). General anthropometric measurements were derived from the health examination survey: height $(\mathrm{cm})$, weight $(\mathrm{kg})$, waist circumference (WC, $\mathrm{cm}$ ), and body mass index (BMI, $\mathrm{kg} / \mathrm{m}^{2}$ ).

Data for general health behavior, such as smoking status, alcohol consumption, and exercise, were obtained from via self-reported questionnaires. The percentage of current smokers who were currently smoking was determined. Heavy drinking status was defined as the ingestion of $\geq 30.0 \mathrm{~g}$ alcohol/day. Exercise was defined as strenuous physical activity at least three times per week or moderate physical activity performed at least five times per week.

Oral health status was established according to the data from a self-administered health interview and health examination. The following parameters were identified: oral pain over the past year, total number of retained teeth, previous orthodontic treatment, any prosthetic appliances, and treatment of periodontal tissues. Oral hygiene behaviors was evaluated by examining the frequency of tooth brushing and the use of oral hygiene products, such as dental floss, interdental brushes, mouth rinse, electronic toothbrushes, water picks, tongue cleaners, specialized tooth brushes for dental implants, and denture care appliances.

The following additionally information was also obtained: glycated hemoglobin ( $\mathrm{HbAic})$ [2], TC, high density lipoprotein cholesterol (HDL-C), low density lipoprotein cholesterol, and triglyceride (TG) levels, and time since diagnosis of DM.

\section{Statistical analysis}

Subjects were divided into two groups, according to the presence or absence of DM. Each group was then divided into a non-periodontitis group and a periodontitis group, according to the CPI value. Continuous variables are presented as means and standard deviations. Categorical variables are presented as percentages. Subgroups were compared to each other: one-way analysis of variance and chi-square tests for mean values of continuous variables and percentages for categorical variables. Variables with $p<0.25$ in the univariate test were selected for the multivariate model, along with age. Statistically significant variables were re-analyzed by multiple logistic regression analysis, to evaluate the odds ratio (OR) and 95\% confidence interval (CI).

In order to minimize bias in the presentation of prevalence rates, complex sampling was applied to calculate the power of each participant (sample weight) as a representative of the Korean population. Statistical analysis was performed using Stata version 12.0 (Stata Co., College Station, TX, USA) and $p$ values of 0.05 or less were considered statistically significant.

\section{RESULTS}

The total number of adults ( $\geq 3$ o years old) with available data for both DM and periodontal status was 4,477. Sample demographics, expressed as mean, were as follows: age, 49.8 years; weight, $64.5 \mathrm{~kg}$; BMI, 24 kg/m²; WC, 82.1 $\mathrm{cm}$; number of teeth, 24.9.

\section{Prevalence of periodontitis according to the pres- ence of DM}

Analysis of the total study population according to periodontitis only, found that subjects with periodontitis were older, had a higher BMI and WC, and fewer teeth, compared to subjects without periodontitis. The DM prevalence of the periodontitis group was $18.7 \%$, which was significantly higher than that of the non-periodontitis group ( $9.0 \%, p<0.001)$.

However, when the sample was divided into four groups, according to the presence of DM and periodontitis, the prevalence of periodontitis, adjusted according to sampling weights for the Korean population, was $43.7 \%$ in the DM group and $25 \%$ in the non-DM group $(p<0.001)$ (Table 1). 
Table 1. Prevalence of periodontitis and characteristics of the subjects according to diabetes and periodontitis

\begin{tabular}{|c|c|c|c|c|c|c|}
\hline \multirow{2}{*}{ Characteristic } & \multicolumn{3}{|c|}{ Non-DM periodontitis } & \multicolumn{3}{|c|}{ DM periodontitis } \\
\hline & $(-)(\mathrm{n}=2,855)$ & $(+)(n=1,005)$ & $p$ value & $(-)(n=350)$ & $(+)(n=267)$ & $p$ value \\
\hline Prevalence & - & $25(1.2)$ & & - & $43.7(1.2)$ & $<0.001$ \\
\hline \multicolumn{7}{|l|}{ Sociodemographic characteristic } \\
\hline Age, yr & $46.9 \pm 0.3$ & $53 \cdot 5 \pm 0.6$ & $<0.001$ & $58.4 \pm 0.8$ & $60.7 \pm 0.9$ & 0.069 \\
\hline Male sex & $45 \cdot 6(1.1)$ & $60.9(1.9)$ & $<0.001$ & $46.4(3.3)$ & $58.1(3.1)$ & 0.012 \\
\hline Education, $\geq$ middle school & $85 \cdot 3(1)$ & $72(2)$ & $<0.001$ & $67(3.2)$ & $56.4(4.1)$ & 0.044 \\
\hline Household income, $<25 \%$ & $10.7(0.9)$ & $19(1.8)$ & $<0.001$ & $24.9(2.5)$ & $32.7(3.7)$ & 0.071 \\
\hline Habitation, rural & $15.9(2.7)$ & $28.2(4.6)$ & $<0.001$ & $22.2(4.2)$ & $29.6(6.5)$ & 0.154 \\
\hline Spouse, yes & $88.8(0.8)$ & $84.8(1.6)$ & 0.007 & $83.4(2.3)$ & $81.3(3)$ & 0.556 \\
\hline \multicolumn{7}{|l|}{ Nutrition } \\
\hline Energy intake, kcal/day & $2,018 \pm 20.9$ & $2,081 \cdot 4 \pm 44 \cdot 3$ & 0.183 & $1,846.8 \pm 60$ & $1,812.1 \pm 61.4$ & 0.699 \\
\hline Protein, g/day & $15.0 \pm 0.1$ & $14.4 \pm 0.2$ & 0.015 & $14.8 \pm 0.3$ & $14.6 \pm 0.4$ & 0.723 \\
\hline \multicolumn{7}{|l|}{ Systemic conditions } \\
\hline Hypertension & $24.8(1.2)$ & $32.2(2)$ & $<0.001$ & $57.2(3.4)$ & $62.6(4)$ & 0.285 \\
\hline Hypercholesterolemia & $13.3(0.8)$ & $14.1(1.3)$ & 0.564 & $29.3(3.1)$ & $28.3(3)$ & 0.815 \\
\hline \multicolumn{7}{|l|}{ Mental health } \\
\hline Stress perception & $26.4(1.1)$ & $22.3(1.8)$ & 0.067 & $25.9(3.2)$ & $23.1(3.2)$ & 0.537 \\
\hline Depression & $13.4(0.7)$ & $13.1(1.3)$ & 0.839 & $15(2.5)$ & $13.8(2.9)$ & 0.760 \\
\hline Thought of committing suicide & $11.9(0.7)$ & $13.2(1.3)$ & 0.365 & $18.1(2.8)$ & $14.5(2.9)$ & 0.383 \\
\hline \multicolumn{7}{|l|}{ Anthropometry } \\
\hline Height, cm & $163.7 \pm 0.2$ & $163.8 \pm 0.4$ & 0.923 & $160.6 \pm 0.6$ & $161.7 \pm 0.7$ & 0.242 \\
\hline Weight, kg & $63.9 \pm 0.3$ & $65 \pm 0.5$ & 0.050 & $65.9 \pm 0.8$ & $66.9 \pm 1.3$ & 0.532 \\
\hline $\mathrm{WC}, \mathrm{cm}$ & $80.7 \pm 0.3$ & $83.2 \pm 0.4$ & $<0.001$ & $86.9 \pm 0.7$ & $88.3 \pm 1$ & 0.289 \\
\hline $\mathrm{BMI}, \mathrm{kg} / \mathrm{m}^{2}$ & $23.8 \pm 0.1$ & $24.2 \pm 0.1$ & 0.011 & $25 \cdot 5 \pm 0.2$ & $25.5 \pm 0.5$ & 0.955 \\
\hline \multicolumn{7}{|l|}{ General health behaviors } \\
\hline Current smoker & $20.3(1.1)$ & $30.1(2.2)$ & $<0.001$ & $16.8(2.5)$ & $26.3(3.2)$ & 0.019 \\
\hline Alcohol, $\geq 30.0 \mathrm{~g} /$ day & $18.8(1)$ & $22.3(1.9)$ & 0.102 & $17 \cdot 4(3)$ & $22.3(3.6)$ & 0.254 \\
\hline Exercise, $\geq$ moderate & $16.8(0.9)$ & $16.9(1.6)$ & 0.977 & $13.6(2.1)$ & $12.8(2.4)$ & 0.823 \\
\hline \multicolumn{7}{|l|}{ Oral health } \\
\hline Oral pain (during the year) & $34(1.3)$ & $45 \cdot 4(2.1)$ & $<0.001$ & $31.5(3.2)$ & $50.4(3.6)$ & $<0.001$ \\
\hline No. of retained teeth & $25.7 \pm 0.1$ & $23.9 \pm 0.2$ & $<0.001$ & $22 \pm 0.5$ & $21.9 \pm 0.5$ & 0.919 \\
\hline Orthodontic treatment, yes & $4.2(0.5)$ & $1.8(0.7)$ & 0.033 & $1.6(1.2)$ & $0.8(0.6)$ & 0.486 \\
\hline Prosthetic treatment, yes & $33.7(1.1)$ & $50.2(2.2)$ & $<0.001$ & $57 \cdot 5(3 \cdot 3)$ & $5^{8}(4.1)$ & 0.984 \\
\hline Periodontal treatment, yes & $8.1(0.6)$ & $15.1(1.4)$ & $<0.001$ & $13.1(2.4)$ & $12(2.5)$ & 0.757 \\
\hline \multicolumn{7}{|l|}{ Oral health behaviors } \\
\hline Tooth brushing (frequency), time/day & & & $<0.001$ & & & 0.024 \\
\hline$\leq 1$ & $9.1(0.8)$ & $15 \cdot 5(1.4)$ & & $12(2.2)$ & $22.3(3.2)$ & \\
\hline Twice & $37 \cdot 4(1.2)$ & $47.1(2.1)$ & & $42.8(3.4)$ & $42.6(3.7)$ & \\
\hline$\geq 2$ & $53.5(1.2)$ & $37.4(2.2)$ & & $45.2(3.5)$ & $35.1(3.9)$ & \\
\hline Oral hygiene product & $51(1.3)$ & $43.6(2)$ & 0.003 & $42.5(3 \cdot 3)$ & $27 \cdot 5(3 \cdot 3)$ & 0.002 \\
\hline
\end{tabular}

Values are presented as number $(\%)$ or mean \pm SD. Household income: monthly average family equivalent income. DM, diabetes mellitus; WC, waist circumference; BMI, body mass index. 
Table 2. Laboratory values in accordance with diabetes and periodontitis

\begin{tabular}{|c|c|c|c|c|c|c|}
\hline \multirow{2}{*}{ Variable } & \multicolumn{3}{|c|}{ Non-DM periodontitis } & \multicolumn{3}{|c|}{ DM periodontitis } \\
\hline & $(-)(n=2,855)$ & $(+)(n=1,005)$ & $p$ value & $(-)(n=350)$ & $(+)(n=267)$ & $p$ value \\
\hline $\mathrm{HbAic}, \%$ & $5 \cdot 5 \pm 0.0$ & $5.6 \pm 0.02$ & $<0.001$ & $7 \cdot 3 \pm 0.1$ & $7.4 \pm 0.1$ & 0.543 \\
\hline $\mathrm{TC}, \mathrm{mg} / \mathrm{dL}$ & $192.2 \pm 1$ & $193.8 \pm 1.4$ & 0.334 & $190.3 \pm 3.7$ & $189.3 \pm 3.2$ & 0.838 \\
\hline HDL-C, mg/dL & $52.4 \pm 0.3$ & $49.3 \pm 0.5$ & $<0.001$ & $47 \cdot 3 \pm 0.8$ & $45 \cdot 4 \pm 0.8$ & 0.068 \\
\hline LDL-C, mg/dL & $114.4 \pm 0.8$ & $116.3 \pm 1.2$ & 0.167 & $105.8 \pm 2.8$ & $109.4 \pm 2.9$ & 0.356 \\
\hline $\mathrm{TG}, \mathrm{mg} / \mathrm{dL}^{\mathrm{a}}$ & $108.8(105.9-111.9)$ & $125.9(120.3-131.8)$ & $<0.001$ & $158.1(144.9-172.6)$ & $152.5(139.4-166.9)$ & 0.566 \\
\hline Anti-HBP medication & $23.4(1.2)$ & $34.4(2.5)$ & $<0.001$ & $42.2(3.8)$ & $45 \cdot 7(4 \cdot 3)$ & 0.506 \\
\hline Anti-lipid medication & $23.4(1.2)$ & $34.2(2.4)$ & $<0.001$ & $41.4(3.7)$ & $45.8(4.3)$ & 0.413 \\
\hline DM duration, yr & - & - & - & $6.2 \pm 0.6$ & $5.8 \pm 0.5$ & 0.639 \\
\hline
\end{tabular}

Values are presented as mean \pm SE or frequency (\%).

DM, diabetes mellitus; HbA1c, glycated hemoglobin; TC, total cholesterol; HDL-C, high density lipoprotein cholesterol; LDL-C, low density lipoprotein cholesterol; TG, triglyceride; HBP, high blood pressure.

aTriglyceride level is presented as geometric mean ( $95 \%$ confidence interval).

\section{Risk factors of periodontitis in subjects without DM}

Among subjects without diabetes $(\mathrm{n}=3,860)$, those with periodontitis were more likely to be male, older age, with lower level of education, lower income, rural habitation, and no spouse. The periodontitis group had a lower daily protein intake, higher BP, weight, WC, and BMI, and a greater proportion of current smokers, compared to the group without periodontitis.

The periodontitis group also had a higher proportion of subjects with experience of oral pain, fewer retained teeth, less frequent tooth brushing, and less frequent use of oral hygiene products compared to subjects without periodontitis. The group also had a history of prosthetic and periodontal treatment and less orthodontic treatment history (Table 1).

Subjects with periodontitis had higher levels of $\mathrm{HbAlc}$ and TGs, lower levels of HDL-C, and increased frequency antihypertension and antidyslipidemia medication compared with those without periodontitis (Table 2).

\section{Risk factors of periodontitis in subjects with DM}

In the DM group, comparison between subjects with and without periodontitis found that those with periodontitis were more likely to be male, current smokers, have a higher level of education, and experience more severe, oral pain. Tooth brushing and use of oral hygiene products was also less frequent (Table 1).

There was also no significant difference in laboratory tests, including $\mathrm{HbA1c}$, lipid profiles, and DM duration, between the two groups (Table 2).

\section{Factors associated with periodontitis according to the presence of DM}

Logistic regression analysis found that age, male sex, urban habitation, WC, current smoking status, experience of oral pain, and history of periodontal treatment history were associated with periodontitis in subjects without DM. Conversely, frequent of tooth brushing was inversely associated with periodontitis (OR, 0.777; 95\% CI, 0.671 to 0.899 ) (Table 3).

In subjects with DM, age, sex, habitation in urban area, WC, history of periodontal treatment, and frequency of tooth brushing were not associated with periodontitis. Only two factors, current smoking status (OR, 1.939; 95\% $\mathrm{CI}, 1.003$ to 3.751) and experience of oral pain experience during the year (OR, 2.383; 95\% CI, 1.460 to 3.888) were positively associated with periodontitis. Furthermore, use of an oral hygiene product was inversely associated with periodontitis (OR, 0.505; 95\% CI, 0.309 to 0.826 ) (Table 4).

\section{DISCUSSION}

This study investigated risk factors for periodontitis, according to the presence of DM, in representative samples of Korean adults ( $\geq 30$ years of age) identified from the KNHANES database.

Using the data from KNHANES 2012 to 2013, Hong et al. [9] demonstrated that the weighted prevalence of periodontitis among the Korean diabetic population $(\geq$ 
Table 3. Multiple logistic regression analysis of periodontitis risk factors in adults without diabetes mellitus

\begin{tabular}{|c|c|c|}
\hline Variable & OR $(95 \% \mathrm{CI})$ & $p$ value \\
\hline \multicolumn{3}{|l|}{ Sociodemographic characteristic } \\
\hline Age, yr & $1.035(1.025-1.046)$ & $<0.001$ \\
\hline Male sex & $1.644(1.269-2.131)$ & $<0.001$ \\
\hline Education, $\geq$ middle school & $0.873(0.612-1.246)$ & 0.455 \\
\hline Household income, $<25 \%$ & $1.005(0.704-1.433)$ & 0.980 \\
\hline Habitation, urban & $1.737(1.229-2.455)$ & 0.002 \\
\hline \multicolumn{3}{|l|}{ Mental health } \\
\hline Stress perception & $0.877(0.677-1.136)$ & 0.320 \\
\hline \multicolumn{3}{|l|}{ Anthropometric measurement } \\
\hline Waist circumference, $\mathrm{cm}$ & $1.014(1.001-1.028)$ & 0.037 \\
\hline \multicolumn{3}{|l|}{ General health behavior } \\
\hline Current smoker & $1.583(1.215-2.062)$ & $<0.001$ \\
\hline Alcohol consumption, $\geq 30.0 \mathrm{~g} /$ day & $0.904(0.670-1.220)$ & 0.510 \\
\hline \multicolumn{3}{|l|}{ Oral health } \\
\hline Oral pain (during the year) & $1.486(1.186-1.861)$ & $<0.001$ \\
\hline No. of retained teeth & $1.007(0.984-1.030)$ & 0.549 \\
\hline Orthodontic treatment, yes & $0.720(0.311-1.670)$ & 0.445 \\
\hline Prosthetic treatment, yes & $1.168\left(0.905^{-1.507)}\right.$ & 0.234 \\
\hline Periodontal treatment, yes & $1.763(1.307-2.379)$ & $<0.001$ \\
\hline \multicolumn{3}{|l|}{ Oral health behavior } \\
\hline Tooth brushing (frequency) & $0.777(0.671-0.899)$ & $<0.001$ \\
\hline Oral hygiene product & $1.015(0.812-1.267)$ & 0.897 \\
\hline
\end{tabular}

OR, odds ratio; CI, confidence interval.

Table 4. Multiple logistic regression analysis of periodontitis risk factors in adults with diabetes mellitus

\begin{tabular}{|c|c|c|}
\hline Variable & OR (95\% CI) & $p$ value \\
\hline \multicolumn{3}{|c|}{ Sociodemographic characteristic } \\
\hline Age, yr & $1.017(0.992-1.042)$ & 0.194 \\
\hline Female sex & $0.900(0.524-1.545)$ & 0.703 \\
\hline Education, $\geq$ middle school & $0.884(0.467-1.673)$ & 0.704 \\
\hline Household income, $<25 \%$ & $0.885(0.497-1.575)$ & 0.677 \\
\hline Habitation, urban & $1.250(0.687-2.727)$ & 0.465 \\
\hline \multicolumn{3}{|l|}{ General health behavior } \\
\hline Current smoker & $1.939(1.003-3.751)$ & 0.049 \\
\hline \multicolumn{3}{|l|}{ Oral health } \\
\hline Oral pain (during the year) & $2.383(1.460-3.888)$ & $<0.001$ \\
\hline \multicolumn{3}{|l|}{ Oral health behavior } \\
\hline Tooth brushing (frequency) & $0.718(0.486-1.062)$ & 0.098 \\
\hline Oral hygiene product & $0.505(0.309-0.826)$ & 0.007 \\
\hline
\end{tabular}

OR, odds ratio; CI, confidence interval. 
19 years) and adult population ( $\geq 19$ years) were $46.7 \%$ and $24.8 \%$, respectively. Furthermore, a study involving United States dentate adults aged $\geq 30$ years, found a periodontitis prevalence of $47 \%$ between 2009 and 2010 [13]. In the present study, the prevalence of periodontitis in adults with $\mathrm{DM}$ ( $\geq 30$ years) was $43.7 \%$.

The difference in periodontitis prevalence between our study and previous research using KNHANES can be explained by variation ( $20.7 \%$ for 2012 vs. $25.2 \%$ for 2013) of the prevalence of periodontitis in the Korean population and by differences in the definition of subject age group categories.

Adults, aged $\geq 30$ years, are the focus of increased attention as potential orthodontic patients. Among subjects without DM, those with periodontitis had fewer retained teeth, less experience of orthodontic treatment but a higher prevalence of both prosthetic treatment and periodontal treatment. They are more likely to be of male sex, with lower education, lower income, and rural habitation without spouse, compared with those without periodontitis; however, HDL-C was significantly lower in adults with periodontitis compared with those without periodontitis.

Conversely, among subjects with DM, there was no association between periodontitis and the following variables: laboratory values, age, household income, living with spouse, number of residual teeth, and experience of orthodontic treatment, prosthetic treatment and periodontal treatment. There was a significant association between periodontitis and the following variables such as male sex, higher levels of education, current smoking status, oral pain, less frequent tooth brushing, and less frequent use of oral hygiene product. In DM periodontitis, one mechanism explaining periodontal tissue destruction is that advanced glycation end products resulting from hyperglycemia leads to the activation of macrophages, producing inflammatory cytokines and reactive oxygen species that increase the periodontal soft and hard tissue destruction [16].

Approximately $25 \%$ of subjects without DM had periodontitis while $47.3 \%$ of those with DM had periodontitis, suggesting that it is clinically important to identify and compare risk factors for periodontitis between the two groups. The manifestation and progression of periodontitis is influenced by a wide range of factors, including demographic characteristics of subjects, so- cial and behavioral factors, systemic factors, genetic factors, microbial composition of dental plaques, and other emerging risk factors [17]. Although, HbAic level was not different among subjects with DM, HbAic level of subjects with periodontitis was significantly higher than that of those without periodontitis among subjects without DM, suggesting a bidirectional relationship between periodontitis and hyperglycemia, with periodontitis inducing hyperglycemia and hyperglycemia increasing the risk of periodontitis.

As with $\mathrm{HbAic}$, WC and BMI of subjects with periodontitis were significantly higher than that of those without periodontitis among subjects without DM. In our study, WC increased the risk of periodontitis by 1.014-fold in subjects without DM. The underlying mechanisms between obesity and periodontitis are not well defined; however, adipose-tissue-derived-cytokines and hormones may play a key role.

We identified a range of factors for periodontitis, according to the presence of DM. In subjects without DM, the risk factors for periodontitis were comparable to previous research (Table 3) [5,9,13]. However, most factors associated with periodontitis in subjects without DM had no significant correlation in subjects with DM except current smoking, experience of oral pain, and use of hygiene products (Table 4). Therefore, if subjects are suffering from DM, even though they are young adults or female who are very interested in dental/orthodontic treatment [12], periodontitis should be screened for and controlled.

Epidemiologic studies have shown increased periodontal disease among older age groups compared with younger age groups $[18,19]$. However, periodontal disease in relatively healthy elderly persons is not caused by the aging process alone [2o]. In our study, the average age of subjects with periodontitis was significantly older than that of those without periodontitis among subjects without DM ( $53.5 \pm 0.6$ vs. $46.9 \pm 0.3, p<0.001)$, while the age was not statistically different among subjects with $\operatorname{DM}(60.7 \pm 0.9$ years vs. $58.4 \pm 0.8$ years, $p=0.069)$.

The association between sex and periodontal disease is controversial. In the present study, among subjects without DM, the proportion of male subjects was higher in the periodontitis group than in the group without periodontitis $(60.9 \%$ vs. $45.6 \%, p<0.001)$ and male sex was significantly associated with periodontitis (OR, 1.644; 95\% CI, 1.269 to 2.131). Clinical attachment loss and bone loss 
were significantly increased in male subjects [18,21]. However, among subjects with DM, there was no difference in the proportion of male subjects between those with periodontitis and those without periodontitis and the periodontitis ORs according to sex (male) was not significant (Table 4). Considering periodontitis as one of the many consequences of chronic systemic inflammation associated with insulin resistance $[22,23]$, insulin-resistant men were found to be more likely to have periodontitis compared to their insulin-sensitive counterparts [24].

Smoking is a well-recognized as a risk factor for periodontal disease [18]. One study showed that smokers were 2.7 times more likely to have moderate to advanced periodontal disease, compared with non-smokers [25]. Significant associations between smoking and both attachment loss and alveolar bone loss have been found $[18,21]$. Tobacco smokers are at an increased risk of periodontal disease, and tooth loss [26]. It is clear that smoking affects the inflammatory and immune responses, as well as the microvasculature [27]. Smoking has a significant impact on the periodontal health of Korean adults; however, the combined effect of smoking and diabetes had no significant impact [28]. In the present study, smoking was associated with periodontitis in adults without DM (OR, 1.583; 95\% CI, 1.215 to 2.062 ) and with $\operatorname{DM}(\mathrm{OR}, 1.939 ; 95 \% \mathrm{CI}, 1.003$ to 3.751).

Patients with type 2 DM may have greater requirements for periodontal treatment than the non-diabetic population; however, baseline glycemic control, duration of diabetes, and smoking were not associated with treatment response [29]. In a further study, smokers were found to have less favorable results following nonsurgical periodontal therapy, specifically less reduction in probing depth [30].

A systematic review and meta-analysis concluded that infrequent tooth brushing was associated with severe forms of periodontal disease [31]. However, tooth brushing is for cleaning teeth surfaces, while oral hygiene products are designed to reach the specific subgingival tissues, such as interdental areas, the gingival sulcus, and peri-implant tissues. DM may have a compounding effect on the periodontium fluid dynamics in periodontal disease [32]. Furthermore, the inflamed and ulcerated subgingival epithelium in periodontitis allows for entry of oral bacteria and/or bacterial components into the blood stream [33].
In our study, a lower frequency of tooth brushing was a risk factor for periodontitis in subjects without DM (OR, 0.777; 95\% CI, 0.671 to $0.899 ; p<0.001$ ). This did not apply to subjects with DM. Interestingly, using oral hygiene products reduces periodontitis in subjects with $\mathrm{DM}$ (OR, 0.505; 95\% CI, 0.309 to $0.826 ; p=0.007$ ), but not in those without DM. Therefore, oral hygiene products, such as dental floss, interdental brushes, mouth rinse, electronic toothbrushes, water picks, tongue cleaners, and specialized tooth brushes for dental implants and dentures should be recommended for subjects with DM. Oral hygiene products generally act on specific areas such as interdental sulcus, missed by tooth brushing, which are very effective for cleaning deep periodontal pockets.

There were a number of limitations of our study. First, CPI is a simple way of evaluating periodontal status in a community survey, although there is a high chance of subjective over- or under-estimation for periodontal pocket. Second, the mean HbArc level was analyzed according to the presence of DM and periodontitis; however, this does not indicate whether the blood glucose levels of the DM patients were adequately managed. Third, the severity of periodontitis was not considered.

Increasing numbers of adults are likely to seek orthodontic treatment. Adult orthodontic treatment has a role in providing complete rehabilitation of both appearance and function, if the patient with periodontitis responds well to periodontal therapy [8]. Also, the prevalence of $\mathrm{DM}$ is increasing in Korea. For orthodontic treatment, well-controlled DM is not a contraindication [34]. In adults with active periodontitis, however, patients with symptoms of DM should always be referred to physician for avoiding uncontrolled or poorly controlled DM.

\section{KEY MESSAGE}

1. The population with diabetes mellitus (DM) has an increased risk of periodontitis, compared to those without DM.

2. Current smoking status and a 1-year history of oral pain are associated with an increased risk of periodontitis in the population with DM.

3. Conversely, use of oral hygiene products can reduce the prevalence of periodontal disease in the population with DM. 


\section{Conflict of interest}

No potential conflict of interest relevant to this article was reported.

\section{REFERENCES}

1. Zhou X, Zhang W, Liu X, Zhang W, Li Y. Interrelationship between diabetes and periodontitis: role of hyperlipidemia. Arch Oral Biol 2015;60:667-674.

2. Jeon JY, Ko SH, Kwon HS, et al. Prevalence of diabetes and prediabetes according to fasting plasma glucose and HbA1c. Diabetes Metab J 2013;37:349-357.

3. Preshaw PM, Bissett SM. Periodontitis: oral complication of diabetes. Endocrinol Metab Clin North Am 2013;42:849867.

4. Mealey BL, Ocampo GL. Diabetes mellitus and periodontal disease. Periodontol 2000 2007;44:127-153.

5. Artese HP, Foz AM, Rabelo Mde S, et al. Periodontal therapy and systemic inflammation in type 2 diabetes mellitus: a meta-analysis. PLoS One 2015;10:e0128344.

6. Poplawska-Kita A, Siewko K, Szpak P, et al. Association between type 1 diabetes and periodontal health. Adv Med Sci 2014;59:126-131.

7. Das M, Upadhyaya V, Ramachandra SS, Jithendra KD. Periodontal treatment needs in diabetic and non-diabetic individuals: a case-control study. Indian J Dent Res 2011;22:291-294.

8. Ong MM, Wang HL. Periodontic and orthodontic treatment in adults. Am J Orthod Dentofacial Orthop 2002;122:420-428.

9. Hong JW, Noh JH, Kim DJ. The prevalence and associated factors of periodontitis according to fasting plasma glucose in the Korean adults: the 2012-2013 Korea National Health and Nutrition Examination Survey. Medicine (Baltimore) 2016;95:e3226.

10. Williams RC. Periodontal disease. N Engl J Med 1990;322:373-382.

11. Jimenez M, Hu FB, Marino M, Li Y, Joshipura KJ. Type 2 diabetes mellitus and 20 year incidence of periodontitis and tooth loss. Diabetes Res Clin Pract 2012;98:494-500.

12. Christensen L, Luther F. Adults seeking orthodontic treatment: expectations, periodontal and TMD issues. $\mathrm{Br}$ Dent J 2015;218:111-117.

13. Eke PI, Dye BA, Wei L, et al. Update on prevalence of periodontitis in adults in the United States: NHANES 2009 to
2012. J Periodontol 2015;86:611-622.

14. Korean National Health and Nutrition Examination Survey. Guideline for survey data [Internet]. Osong (KR): Korean National Health and Nutrition Examination Survey, c2015 [cited 2016 Aug 8]. Available from: https://knhanes. cdc.go.kr/knhanes/.

15. Kweon S, Kim Y, Jang MJ, et al. Data resource profile: the Korea National Health and Nutrition Examination Survey (KNHANES). Int J Epidemiol 2014;43:69-77.

16. Taylor JJ, Preshaw PM, Lalla E. A review of the evidence for pathogenic mechanisms that may link periodontitis and diabetes. J Periodontol 2013;84(4 Suppl):S113-S134.

17. Nunn ME. Understanding the etiology of periodontitis: an overview of periodontal risk factors. Periodontol 2000 2003;32:11-23.

18. Grossi SG, Zambon JJ, Ho AW, et al. Assessment of risk for periodontal disease. I. Risk indicators for attachment loss. J Periodontol 1994;65:260-267.

19. Streckfus CF, Parsell DE, Streckfus JE, Pennington W, Johnson RB. Relationship between oral alveolar bone loss and aging among African-American and Caucasian individuals. Gerontology 1999;45:110-114.

20. Ajwani S, Ainamo A. Periodontal conditions among the old elderly: five-year longitudinal study. Spec Care Dentist 2001;21:45-51.

21. Grossi SG, Genco RJ, Machtei EE, et al. Assessment of risk for periodontal disease. II. Risk indicators for alveolar bone loss. J Periodontol 1995;66:23-29.

22. Lee JB, Yi HY, Bae KH. The association between periodontitis and dyslipidemia based on the Fourth Korea National Health and Nutrition Examination Survey. J Clin Periodontol 2013;40:437-442.

23. Nibali L, Tatarakis N, Needleman I, et al. Clinical review: association between metabolic syndrome and periodontitis: a systematic review and meta-analysis. J Clin Endocrinol Metab 2013;98:913-920.

24. Lim SG, Han K, Kim HA, et al. Association between insulin resistance and periodontitis in Korean adults. J Clin Periodontol 2014;41:121-130.

25. Haber J, Kent RL. Cigarette smoking in a periodontal practice. J Periodontol 1992;63:100-106.

26. Krall EA, Dawson-Hughes B, Garvey AJ, Garcia RI. Smoking, smoking cessation, and tooth loss. J Dent Res 1997;76:1653-1659.

27. Dietrich T, Bernimoulin JP, Glynn RJ. The effect of cigarette smoking on gingival bleeding. J Periodontol 
2004;75:16-22.

28. Han DH, Lim S, Kim JB. The association of smoking and diabetes with periodontitis in a Korean population. J Periodontol 2012;83:1397-1406.

29. Michalowicz BS, Hyman L, Hou W, et al. Factors associated with the clinical response to nonsurgical periodontal therapy in people with type 2 diabetes mellitus. J Am Dent Assoc 2014;145:1227-1239.

30. Garcia RI. Smokers have less reductions in probing depth than non-smokers following nonsurgical periodontal therapy. Evid Based Dent 2005;6:37-38.

31. Zimmermann H, Zimmermann N, Hagenfeld D, Veile A, Kim TS, Becher $\mathrm{H}$. Is frequency of tooth brushing a risk factor for periodontitis? A systematic review and meta-analysis. Community Dent Oral Epidemiol 2015;43:116-127.

32. Sakallioglu EE, Lutfioglu M, Sakallioglu U, Diraman E, Keskiner I. Fluid dynamics of gingiva in diabetic and systemically healthy periodontitis patients. Arch Oral Biol 2008;53:646-651.

33. Nguyen CM, Kim JW, Quan VH, Nguyen BH, Tran SD. Periodontal associations in cardiovascular diseases: the latest evidence and understanding. J Oral Biol Craniofac Res 2015;5:203-206.

34. Bensch L, Braem M, Van Acker K, Willems G. Orthodontic treatment considerations in patients with diabetes mellitus. Am J Orthod Dentofacial Orthop 2003;123:74-78. 